\title{
ÉDITORIAL
}

\section{Natures, Sciences, Sociétés : propos d'étape}

$\mathrm{L}$ a revue Natures, Sciences, Sociétés a maintenant trois ans révolus. II n'est pas inutile de se livrer à un bilan. Peut-être même ce bilan estil nécessaire pour clarifier une démarche éditoriale qui, à la lumière de l'expérience acquise, nous paraît plus que jamais indispensable, réalisable, novatrice et fructueuse.

Rappelons l'objectif : tracer les voies d'une recherche qui prenne en charge les deux préoccupations majeures de notre temps à travers lesquelles les sociétés humaines contemporaines s'interrogent sur leurs rapports avec les fondements bio-physico-chimiques de leur existence, la question de l'environnement et les problèmes bioéthiques.

La vivacité actuelle des débats autour de ces deux champs de réflexion ne témoigne-t-elle pas de la crise de ces rapports et cette crise n'appelle-t-elle pas des réponses scientifiques adéquates?

॥ est inévitable qu'un tel projet conduise à aborder un nombre fort élevé de thèmes. II suffit pour s'en convaincre de parcourir le sommaire des douze numéros parus durant ces trois années. Et encore, on pourrait énumérer une liste fort longue de thèmes absents de ce panorama et qui mériteraient pourtant d'y figurer. Une telle diversité thématique peut être perçue comme un inconvénient pour un lecteur qui cherche une information précise et systématique dans un domaine bien circonscrit. Mais l'idée centrale qui sous-tend le projet même de notre revue est que les réponses aux questions posées ne viendront pas nécessairement d'une recherche circonscrite à un domaine restreint, mais au contraire de l'exemple trouvé dans un autre champ disciplinaire plus avancé sur le plan de la démarche scientifique. Défense et illustration de la démarche systémique, recherche des acquis et des progrès dans les problématiques et les méthodes transférables d'une thématique à l'autre : telles sont les deux règles d'or de la ligne éditoriale suivie. D'où une volonté constante de la rédaction de mettre en évidence tout ce qui contribue à rendre visible l'unité de la démarche d'analyse ou, au moins, d'ouvrir la voie à des correspondances ou à des convergences possibles. Les textes publiés dans Natures, Sciences, Sociétés apportent bien sûr une information, des données, des conclusions valables pour elles-mêmes sur la question dont ils traitent, mais ils comportent aussi une dimension susceptible d'être de portée générale et par conséquent d'être transposée à d'autres thèmes, objets, questions de recherche ou domaines d'action. A chacun bien sûr de trouver la "substantifique moelle" qui correspond à ses centres d'intérêt, à travers cet exercice de lecture en quelque sorte "décalée" ou "à côté", pourrait-on dire.

Au cœur de ce projet, figurent constamment deux questions. Premièrement, comment penser et conduire sa recherche particulière en ayant à l'esprit cette interrogation englobante et récurrente sur les rapports entre les sociétés humaines, le vivant et la géosphère qui en est à la fois le support et la coquille ? Peut-on se référer à un champ de recherche commun, à une problématique unifiée, qui permettent de situer toutes les recherches en cause les unes par rapports aux autres, de quelqu'horizon qu'elles viennent? Deuxièmement, comment penser et conduire sa recherche dans ces domaines en tenant compte de l'impératif de l'action? Ne peuton pas dégager du croisement des démarches systémique et interdisciplinaire une conception même de l'action qui corresponde aux exigences spécifiques des problèmes abordés et à traiter ?

Les douze livraisons de Natures, Sciences, Sociétés qui correspondent à ses trois premières années d'existence ont d'ores et déjà apporté des contributions multiples à ces questions. Le chantier est vaste et appellera toujours de sérieux efforts de tous les corps de métiers. Sans doute va-t-il falloir dorénavant identifier les questions cruciales à traiter. Nous vous convions à le faire avec nous, en continuant à nous accorder la confiance dont vous nous donnez de plus en plus de preuves. Ainsi s'ouvrira une nouvelle étape et dans la vie de Natures, Sciences, Sociètés et dans l'évolution de la démarche de recherche que nous voulons promouvoir. 